\title{
Comparison of Various Election Algorithms in Distributed System
}

\author{
Priyanka Gupta \\ Computer Science \& Engineering Department, \\ SVITS, Indore \\ Indore (M.P.) India
}

\author{
Rajeev G.Vishwakarma, Ph.D \\ Computer Science \& Engineering Department, \\ SVITS, Indore \\ Indore (M.P.) India
}

\begin{abstract}
This paper present the comparison between the various available algorithms on the basis of their structure, assumptions and the main thing is complexity. We compare the algorithms with our proposed one. We take the message complexity of various algorithms from previous papers. And calculate the message complexity for our newly define approach, and compare the calculated complexity with all the available algorithms complexity. The result shows that our proposed approach is more efficient than the previous approaches also it has less no of message passing during communication.
\end{abstract}

\section{General Terms}

Communication, System, link, network, Algorithm

\section{Keywords}

Processes ,node ,Distributed System , priority

\section{INTRODUCTION}

A Distributed system is an application that executes a collection of protocols to coordinate the actions of multiple processes on a network, such that all components cooperate together to perform a single or small set of related tasks. It is difficult for processes to cooperate with each other because failures of any of the process during communication .In distributed system a single process is choose to control or coordinate the operation of the entire system. So entire system will run smoothly. To decide which process takes this responsibility, there are several algorithms available that give the idea, which process works as coordinator in the system. In this paper we first present the concept of election algorithm in section 2 and in section 3 we take the review of different existing election algorithms and their disadvantages .In section 4 we present our proposed Algorithm and compare it with existing election algorithms. In section 5 we Present Mathematical Description of different Existing Algorithms and In Section 6 comparison table and Section 7 And 8 contains Conclusion and References Respectively.

\section{ELECTION ALGORITHMS}

The election algorithm is an algorithm for choosing a coordinator or leader in the entire system from all the currently available running processes. Since all other processes in the system have to interact with the coordinator ,they all must agree on who the coordinator is.Furthermore, if the coordinator process fails due to any reason e.g. Hardware fail, link fail, etc.,a new coordinator process must be elected to take up the job of the failed coordinator. An election algorithm is used for solving the all above problems

\section{EXISTING ALGORITHMS}

Many distributed election algorithms have been proposed to resolve the problem of leader election. Among all the existing algorithms, the most prominent algorithms are as

a. Bully Algorithm presented by Gracia-Molina in 1982.

b. Improved Bully Election Algorithm in Distributed System presented by A.Arghavani in 2011.

c. Modified Bully Election Algorithm in Distributed Systems presented by M.S.Kordafshari and group.

\subsection{Bully Algorithm by Gracia-Molina}

Bully Algorithm is one of the most promising election algorithms which were presented by Gracia Molina in 1982.The algorithm take the following assumption.

\subsubsection{Assumption.}

$\checkmark$ Each process has a unique number to distinguish them.

$\checkmark \quad$ Each process knows the process number of all other process.

$\checkmark \quad$ In the election, a process with the highest process number is elected as a coordinator.

$\checkmark \quad$ A failed process can rejoin in the system after recovery.

$\checkmark \quad$ The model is timely bounded.

\subsubsection{Algorithm}

The algorithm describe in the following steps.

$\checkmark \quad$ When a process P detects that the coordinator is not responding on time, it initiates an election and send election message to all processes with higher number.

$\checkmark \quad$ If no one other processes respond within time, $\mathrm{P}$ wins the election and becomes coordinator.

$\checkmark \quad$ When a process receives an election message from any lowered number process.

$\checkmark \quad$ The receiver process sends ok back to the sender process.

$\checkmark$ Now the receiver holds an election, unless it is already holding one.

$\checkmark$ And now this process sends the election message to higher number process than itself.

$\checkmark \quad$ All the process gives up except one that is the new coordinator.

$\checkmark \quad$ New coordinator announces itself as a coordinator by sending leader message to all other process in the entire system. 


\subsubsection{Disadvantages.}

Bully algorithm has following disadvantages.

$\checkmark$ It required that every process should know the identity of every other process in the system so it takes very large space in the system.

$\checkmark \quad$ It has high number of message passing during communication which increases heavy traffic the message passing has order o (n2).

\subsection{Modified bully Election Algorithm in Distributed System}

The Modified bully Election Algorithm is presented by M.S. Kordafshari, M.gholipour, M.jahanshahi, A.T.haghighat in 2005.the algorithm resolve the disadvantages of the bully algorithm. The Modified bully algorithm takes the following assumption.

\subsubsection{Assumption.}

This algorithm assumes the same system model as in previously defined bully algorithm. But follow the different procedure.

\subsubsection{Algorithm}

$\checkmark \quad$ When any process $\mathrm{p}$ notices that coordinator is not responding, it initiates an election and send election message to all process with higher priority number.

$\checkmark \quad$ If no process responds, process $\mathrm{P}$ wins the election and becomes new coordinator.

$\checkmark$ Process with the higher priority sends ok message with its priority number to process $\mathrm{P}$.

$\checkmark \quad$ When process $\mathrm{p}$ receive all the response it select the new coordinator with the highest priority number process and sends the grant message to it.

$\checkmark$ Now the coordinator process will broadcast a New coordinator message to all other process and informs itself as a coordinator.

\subsubsection{Disadvantages}

$\checkmark \quad$ A modified algorithm is also time bounded.

$\checkmark \quad$ It is better than bully but also has o (n2) complexity in worst case.

$\checkmark \quad$ It is necessary for all process to know the priority of other.

\subsection{Improved Bully Election Algorithm in Distributed Systems}

This algorithm is presented by A.arghavani, E.ahmadi, A.T.haghighat in 2011. It also overcomes the disadvantages of the original bully. The main concept of this algorithm is that the algorithm declares the new coordinator before actual or current coordinator is crashed. This algorithm also makes some assumption.

\subsubsection{Asuumption}

$\checkmark \quad$ Each process has a unique number to distinguish them.

$\checkmark \quad$ Each process knows the process number of all other process.

$\checkmark \quad$ When any new process enters in the running system. It sends its id to coordinator and coordinator update the list of available process and send in the network. $\checkmark \quad$ There is no time bound during communication.

\subsubsection{Algorithm}

This algorithm selects the coordinator before current coordinator is crashed. So it takes extra stages. In this algorithm before the coordinator is failed, the current coordinator tries to gather information about processes in the system and through the current coordinator, declares the next possible coordinator to the processes. With increasing knowledge and get the id of all other process, a process with the bigger id attempts to execute the bully algorithm.

If the coordinator is failed, each process that notices this failure compares its id with the id which it has received via the coordinator. And select the new coordinator

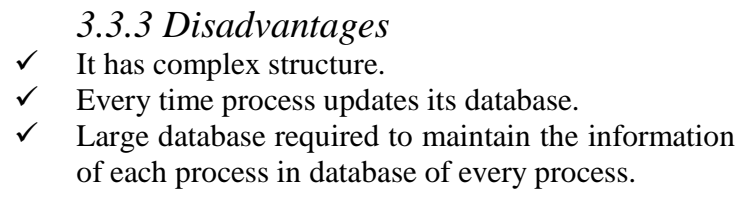

\section{OUR PROPOSED ALGORITHM}

In these algorithm we use several nodes or processes with its own priority no. Initially using the Modified Algorithm a node with the highest priority no. is selected as leader. And then broadcast this new leader information to all other nodes in the network. At any time if any node or process $\mathrm{P}$ in the network finds that leader is crashed, it wait for some time and broadcast leader crash message in the network. So every node in the network receives this leader crash message.

\subsection{Assumption}

$\checkmark \quad$ Each process has a distinct priority number.

$\checkmark$ There is no need to know the priority number of other processes.

$\checkmark \quad$ There is no bound on time.

$\checkmark$ The higher priority process is elected as a leader.

\subsection{Algorithm}

Process run the Election algorithm as follow.

$\checkmark$ When a node or process $\mathrm{P}$ finds that leader is crashed, send Leader Crash message with its own priority number to all processes in the network.

$\checkmark$ Processes that have highest priority no. than the sender's priority no. respond to process $\mathrm{P}$.

$\checkmark \quad$ If process $\mathrm{P}$ doesn't receives any response then $\mathrm{P}$ wins the Election and becomes a leader.

$\checkmark \quad$ If some higher priority no. processes response to process $\mathrm{P}$ with their priority number then process $\mathrm{P}$ will select the coordinator.

$\checkmark$ Process P selects a leader on the basis of highest priority no. among all the responses and then broadcast this new leader information in the network

\subsection{Advantages}

$\checkmark \quad$ Low space required because it's not necessary for every process to know every other process.

$\checkmark \quad$ Less no of message passing during communication

$\checkmark \quad$ Less complex structure. 
Time is not bounded, so no need for fault tolerant mechanism.

\section{MATHEMATICA DESCRIPTION}

$\checkmark$ The Bully algorithm derives the formula for calculating total number of message.

So total no. of messages in bully algorithm is

$\mathrm{N}(\mathrm{r})=(\mathrm{n}-\mathrm{r}+1)(\mathrm{n}-\mathrm{r})+(\mathrm{n}-1)$

$\mathrm{N}=$ Total messages

$\mathrm{n}=$ Total no. of processes

$\mathrm{r}=$ priority no. of processes who find out the crashed coordinator

$\checkmark \quad$ In The Modified bully algorithm the formula is as follow

$\mathrm{N}(\mathrm{r})=2 *(\mathrm{n}-\mathrm{r})+\mathrm{n}$

$\mathrm{N}=$ Total messages

$\mathrm{n}=$ Total no. of processes

$r=$ priority no. of processes who find out the crashed coordinator

$\checkmark$ In Improved algorithm the total no of message passing during communication is calculate by following equation

$\mathrm{N}(\mathrm{r})=\mathrm{m}+(\mathrm{n}-\mathrm{r}+1)(\mathrm{n}-\mathrm{r})+\mathrm{n}$
$\mathrm{N}=$ Total messages

$\mathrm{n}=$ Total no. of processes

$\mathrm{r}=$ priority no. of processes who find out the crashed coordinator

$\mathrm{m}=\mathrm{n} * \mathrm{k}$

$\mathrm{k}=$ no. of times that coordinator sends the biggest found id to processes.

$\checkmark \quad$ In our newly derive approach the total no. of message passing is as

$\mathrm{N}(\mathrm{r})=2(1 \mathrm{~b})+((\mathrm{n}-1)-\mathrm{r})$

$\mathrm{N}=$ Total messages

$\mathrm{n}=$ Total no. of processes

$\mathrm{r}=$ priority no. of processes who find out the crashed coordinator

$1 \mathrm{~b}=$ broadcasting

\section{COMPARISON TABLE}

Table shows that our algorithm is better than existing one. It has less no. of message passing during communication. Also has less complexity than previously defined algorithms.

In the comparison table we calculate the messages for $\mathrm{r}=1$ (lowest priority) for every algorithm.

And in the case of improved bully algorithm the value of $m$ is assume 0 .

The $\mathrm{n}$ denote the node value and N(r) denote the total number of messages required for communication in respective node value.

We calculate the messages for different node value. In this paper we take the value of node from 5 to 10000 .

As shown in the table there is very large difference between

Messages in each algorithm used for communication.

Table 1.Comparison of leader Election Algorithm

\begin{tabular}{|c|c|c|c|c|}
\hline \multirow[b]{2}{*}{$\begin{array}{l}\text { No. of nodes } \\
\text { (n) }\end{array}$} & \multicolumn{4}{|c|}{ Total No. of messages } \\
\hline & $\begin{array}{c}\text { Bully Algorithm } \\
\text { N(r) }\end{array}$ & $\begin{array}{c}\text { Modified Bully } \\
\text { Algorithm } \\
\text { N(r) }\end{array}$ & $\begin{array}{c}\text { Improved Bully Election } \\
\text { Algorithm in Distributed } \\
\text { System } \\
\text { N(r) }\end{array}$ & $\begin{array}{c}\text { Our Proposed } \\
\text { Algorithm } \\
\text { N(r) }\end{array}$ \\
\hline 5 & 24 & 9 & 24 & 5 \\
\hline 50 & 2499 & 148 & 2499 & 50 \\
\hline 100 & 9999 & 298 & 9999 & 100 \\
\hline 150 & 22499 & 498 & 22499 & 150 \\
\hline 200 & 39999 & 598 & 39999 & 200 \\
\hline 500 & 249999 & 1498 & 249999 & 500 \\
\hline 1000 & 999999 & 2998 & 999999 & 1000 \\
\hline 10000 & 99999999 & 29998 & 99999999 & 10000 \\
\hline
\end{tabular}




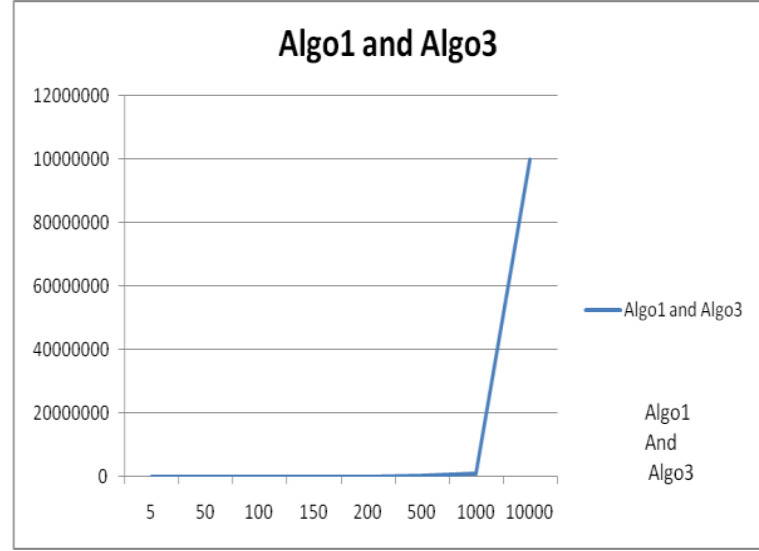

Fig 1.Message Complexity in Bully and Improved Bully

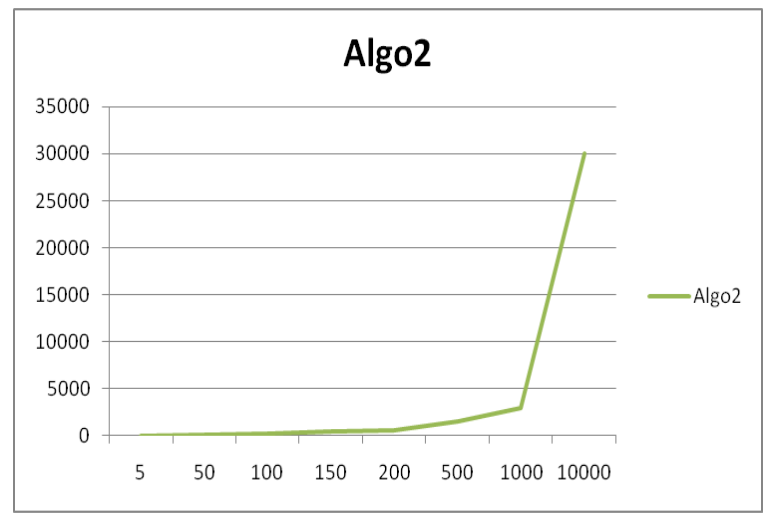

Fig 2.message Complexity in Modified Bully

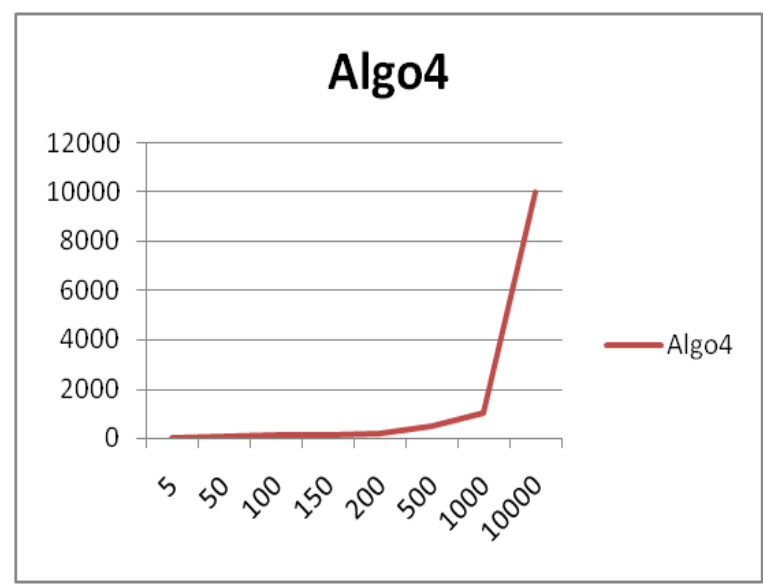

Fig 3.Message Complexity in our Proposed Algorithm

The Above graph shows that our algorithm required less number of message passing during communication for the same node value.

Hence it is proved that our newly proposed algorithm is better than previously defined algorithms

\section{CONCLUSION AND FUTURE WORK}

There are several election algorithms available in Distributed system. In this paper we discuss the concept of some existing algorithms. We take the mathematical analysis of existing algorithm and based on this analysis we realizes some of disadvantages of existing algorithms. To overcome these disadvantages we proposed our new algorithm, and compare our newly proposed algorithm with some of existing algorithms .the result clearly shows that our new algorithm is better than previously defined algorithms .the new algorithm also reduces the no. of message passing in the system. In future we implement this algorithm on different networks and analyze the result of different networks.

\section{REFERENCES}

[1] H. Garcia Molina, "Elections in a Distributed Computing System." IEEE Trans. Comp, 1982, vol.31.

[2] A.Arghavani,E.ahmadi,A.t.Haghihat'Improved Bully Election in Distributed System" Malaysia 2011

[3] N. Fredrickson and N. Lynch, "Electing a Leader in asynchronous Ring.” J.ACM, 1987, vol.34

[4] Quazi Ehsanul Kabir Mamum,Salahuddin Mohammad Masum,Mohammad Abdur Rahim Mustafa"Modified Bully Algorithm for Electing Coordinator in Distributed System" Austria 2004

[5] G. Le Lann, "Distributed systems-Towards a formal approach," Information Processing 77, Amsterdam: North Holland, pp. 155-160, 1977.

[6] S.Park, Y.Kim and J.S.Hwang, "An Efficient Algorithm for Leader-Election in Synchronous Distributed Systems,"1999 IEEE TENCON.

[7] J. Brunekreef, J.-P. Katoen, R. Koymans, and S. Mauw,"Design and analysis of Dynamic leader election protocols in broadcast networks," Distributed Computing, vol.9

[8] Tai Woo Kim , Eui Hong Kim, Joong Kwon Kim “ A Leader Election Algorithm in a Distributed Computing System" IEEE 1995

[9] M.Gholipur, M.S.Kordafshri, M.Jahanshani, A.M.Rahmani “ A New Approach For Election Algorithm in Distributed Systems"IEEE 2009

[10] Mohammad Reza Effat Parvar,Nasser Yazsani, Mehdi EddatParvar, Aresh Dadlani , and Ahmad khonsari ", Improved Algorithms for Leader Electon in Sidtributed Systems" IEEE 2010

[11] M.Gholipur, M.S.Kordafshri, M.Jahanshani, A.M.Rahmani “ Modified Bully Election Algorithm in Distributed Systems" 2005

[12] Mohammad Abdur Rahim, Afroza Nahar " Modified Bully Algorithms Using Election Commission "

[13] Paul Krzyzanowski “ Process Synchronization and Election Algorithm " 2009

[14] Rebecca Ingram, Patrick Shields , jennifier L.Walter “ An Aynchronous Leader Election Algorithm For Distributed Network" 2009

[15] S.Park, Y.Kim and J.S.Hwang, "An Efficient Algorithm for Leader-Election in Synchronous Distributed Systems,"1999 IEEE TENCON 